\title{
The Virgo Cluster
}

\author{
Jeremy Mould ${ }^{1,2}$ \\ ${ }^{1}$ Centre for Astrophysics and Supercomputing, Swinburne University, Hawthorn 3122, \\ Australia \\ ${ }^{2}$ ARC Centre of Excellence for All-sky Astrophysics (CAASTRO) \\ email: jmould@swin.edu.au
}

\begin{abstract}
In the era of precision cosmology, the Virgo cluster takes on a new role in the cosmic distance scale. Its traditional role of testing the consistency of secondary distance indicators is replaced by an ensemble of distance measurements within the Local Supercluster, united by a velocity-field model obtained from a reconstruction based on redshift surveys. The Wilkinson Microwave Anisotropy Probe (WMAP) leads us to see the Hubble constant as one of six parameters in a standard model of cosmology with considerable covariance among parameters. Independent experiments, such as WMAP, the Hubble Space Telescope Key Project on the Extragalactic Distance Scale, and their successors constrain these parameters.
\end{abstract}

Keywords. surveys, galaxies: distances and redshifts

\section{Introduction}

At the height of the 1980s distance-scale controversy, the Virgo cluster had iconic status. Now, following the Las Campanas Virgo Cluster survey (Binggeli et al. 1985) and X-ray imaging, we see Virgo as a concentration of matter to be mapped. Its place has been taken by an ensemble of distance measurements within $z<0.01$, linked by a velocity-field model developed from the 2MAss redshift survey. Some expansion-rate myths are discussed in this review, and how to see past them. We should be aiming to measure $\mathrm{H}_{0}$ to $1 \%$ accuracy.

\section{Virgo galaxy distances}

Fig. 1 has been prepared from the NASA Extragalactic Database (NED) compilation of Virgo distances. Similar results have been obtained by Mei et al. (2007) using the surface brightness fluctuations (SBFs) distance indicator. A map of the cluster in projection is furnished by Bohringer et al. (1994), based on ROSAT observations.

In a dozen years, we have moved from the distance-scale controversy illustrated by Table 1 of Tammann (1999) to a cluster-mapping perspective. It is noteworthy that the Tully-Fisher (TF) relation, which celebrates its $35^{\text {th }}$ anniversary this year, provided the first correct measurement of the distance to Virgo (Mould et al. 1980), $(m-M)_{0}=$ $31.0 \pm 0.1 \mathrm{mag}$. No morphological-type dependence was seen (Aaronson \& Mould 1983). One might ask, what about two galaxies with the same rotational velocity/linewidth $(\Delta V)$ and significantly different bulges? Semi-analytic models show a TF morphologicaltype dependence (C. Tonini et al., in prep.), which can be removed by plotting a new dynamical parameter combining velocity dispersion and $\Delta V$. The reason for the weakness of the TF morphological-type dependence is the strong correlation of galaxy mass with bulge velocity dispersion (Catinella et al. 2012). 


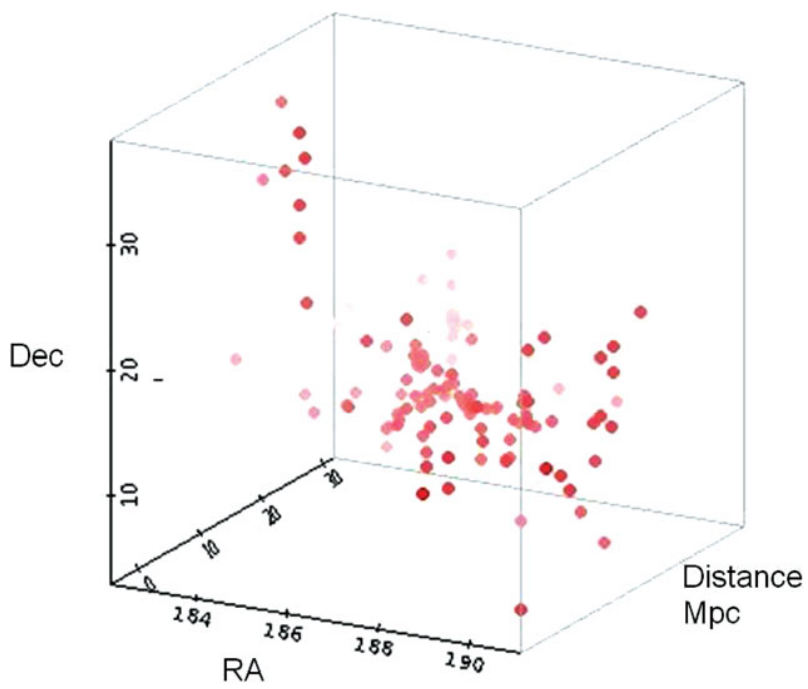

Figure 1. Structure of the Virgo cluster based on the NED 1D database.

Table 1. Virgo cluster distance modulus from various methods (Tammann 1999).

\begin{tabular}{lcl}
\hline Method & $(m-M)_{\text {Virgo }}$ & Hubble type \\
\hline Cepheids & $31.52 \pm 0.21$ & Spiral \\
SNe Ia & $31.39 \pm 0.17$ & Elliptical, Spiral \\
Tully-Fisher & $31.58 \pm 0.24$ & Spiral \\
Globular clusters & $31.67 \pm 0.15$ & Elliptical \\
$D_{n}-\sigma$ & $31.85 \pm 0.19$ & S0, Spiral \\
Novae & $31.46 \pm 0.40$ & Elliptical \\
Mean: & $\mathbf{3 1 . 6 0} \pm \mathbf{0 . 0 8}$ & $(\equiv 20.9 \pm 0.8 \mathrm{Mpc})$ \\
\hline
\end{tabular}

\section{Secondary distance indicators in Virgo}

In the ACS Virgo cluster survey, Mei et al. (2007) provide Hubble Space Telescope (HST)/ACS imaging of 100 early-type galaxies and derive distances to 84 based on application of the SBF technique. Five galaxies have distances $d \approx 23 \mathrm{Mpc}$ and are members of the $\mathrm{W}^{\prime}$ Cloud. For the remaining 79 galaxies, the mean distance is $16.5 \pm 0.1$ (random) \pm 1.1 (systematic) Mpc. The rms distance scatter is $0.6 \pm 0.1 \mathrm{Mpc}$. The backto-front depth of the cluster is $2.4 \pm 0.4 \mathrm{Mpc}$. The M87 (Cluster A) and M49 (Cluster B) subclusters lie at $16.7 \pm 0.2$ and $16.4 \pm 0.2 \mathrm{Mpc}$, respectively. Virgo's early-type galaxies appear to define a slightly triaxial distribution, with axis ratios of (1:0.7:0.5).

Following this, we have the Next Generation Virgo Survey (NGVS; Ferrarese et al. 2012). They anticipate distance errors of $0.13 \mathrm{mag}$ from the NGVS data, corresponding to $\pm 1 \mathrm{Mpc}$. It is possible to study how galaxy properties correlate with the true local environment determined from 3D positions. They expect distances for more than 200 galaxies, allowing spatial segregation by galaxy type.

The M87 distance from an HST colour-magnitude diagram is provided by Bird et al. (2010). A deep $(V, I)$ imaging dataset of M87 with HST's ACS camera resolved its brightest red-giant stars. As a byproduct, they obtained a preliminary measurement of the distance to M87 with the tip of the red giant branch (TRGB) method; the result was $(m-M)_{0}=31.12 \pm 0.14 \mathrm{mag}(d=16.7 \pm 0.9 \mathrm{Mpc})$. 


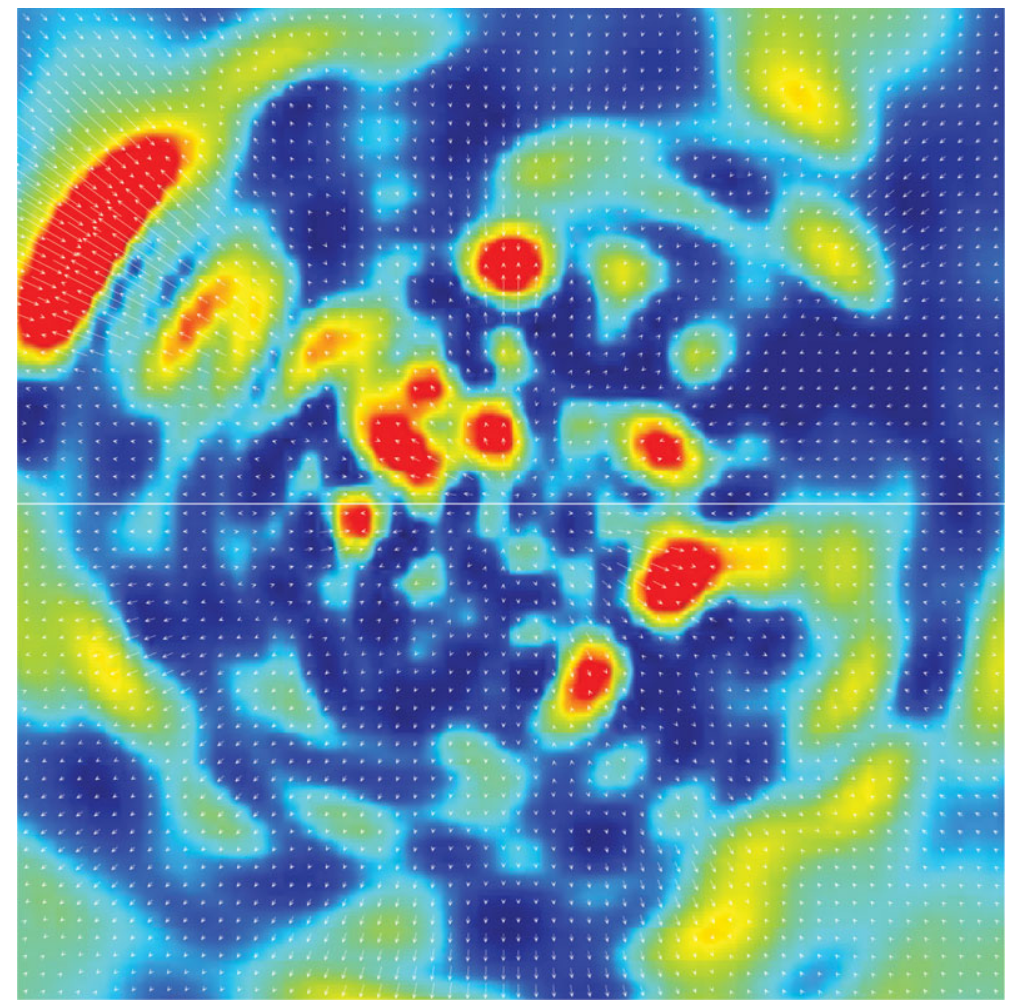

Figure 2. Density field (coloured) in the Supergalactic Plane and the velocity field within 150 Mpc based on 2Mrs (Erdoğdu et al. 2006). We are at the centre (origin). The largest structure shown is the Shapley Supercluster.

For TRGB and Cepheids, Mould \& Sakai (2008, 2009a,b) find that when the TRGB is used as a standard candle, the value of the Hubble constant is the same as when Cepheid stars are used. This finding is in agreement with Tammann (this volume). This confirms the findings of the HST Key Project on the Extragalactic Distance Scale, although that finding is in disagreement with Tammann's.

The globular cluster luminosity function (GCLF) is not reviewed elsewhere in this Symposium. Villegas et al. (2010) studied the GCLF turnover magnitude, $\mu_{z}$, for all galaxies in the ACS Virgo and Fornax cluster surveys. They found $\mu_{z}=(23.51 \pm 0.11)+$ $(0.04 \pm 0.01) M_{z, \mathrm{gal}}$, plus an offset of $\delta(m-M)=0.20 \pm 0.04$ mag for the galaxies in Fornax.

We can also ask if secondary distance indicators continue to be necessary if Cepheids can be found and measured in the Coma cluster. $\dagger$

\section{Replacing Virgo with the ensemble of local supercluster distances}

Fig. 2 shows the velocity field that allows Local Supercluster distances to be used as an ensemble to supplement the Virgo cluster distance in the traditional distance-scale ladder. A similar velocity field has been calculated by Lavaux \& Tully (2010), also from

$\dagger$ Samantha Hoffmann will present these in her $\mathrm{PhD}$ thesis talk at the 2013 winter American Astronomical Society meeting. 
the 2MRs survey. The corresponding distance catalog is compiled from the following sources:

- HST Cepheids;

- EDD (Tully); $\dagger$

- SDSs Fundamental Plane (George);

- Feldman, Watkins, \& Hudson (2010) catalog;

- Nearby supernovae Type Ia (SNe Ia) distances;

- SBF distances (Tonry 2001);

- SFI++ (Springob et al.2007);

- NED database;

One can, however, ask, does mass follow light? Reconstructions are based on the assumption that it does, but chinks in that argument exist in the separation of dark and luminous matter in the Bullet Cluster and in evidence for bulk flows not predicted by 2MRS (Magoulas 2012).

One may also ask about the uncertainties in this velocity field. One answer is consistency with the Lavaux et al. (2010) velocity field which is also based on 2MRs. Comparisons between Erdoğdu et al. (2006) and Lavaux et al. (2010) show a lot of scatter. Another answer is to recompute Erdoğdu's velocities by adding Monte Carlo-style perturbations to it. A third answer is to adopt some heuristic errors scaled to the density of the region of any particular galaxy of interest. For example, in cluster density regions (e.g. Virgo) — where there tend to be few spirals anyway - the uncertainty is as large as the cluster velocity dispersion, because the region is virialized. Indeed, in such regions one is best off using the cluster distance for all galaxies within a projected Mpc or so. Then there are very low-density regions, where computed velocities should be near perfect (modulo the uncertainty in biasing). And there are intermediate-density regions where the uncertainty would be somewhere in between. Future improvements to the velocity field will come from

- more distances: e.g. WALLABY/SkyMapper $₫$ and wnshs/PanSTARRS\|

- deeper redshift surveys: e.g. TAIPAN (UK Schmidt telescope),$\dagger \dagger$ the ongoing $2 \mathrm{MRS}$ (Huchra et al. 2012), and possibly future work at Apache Point Observatory.

- better velocity calculations beyond the linear approximation and employing higher resolution.

\section{Myths}

A long-standing furphyłf is that there is no such thing as the Hubble constant. Density variations make the expansion rate a function of position $H(\theta, \phi, z)$. A recent misunderstanding is that the traditional distance ladder has been replaced by fitting cosmic microwave background (CMB) anisotropies and what they evolve into. The sound horizon is the new standard ruler. A third fundamentalist (or extreme empiricist) view is that $\mathrm{H}_{0}$ from CMB anisotropies is model-dependent and not strictly relevant to measuring the Hubble constant. To these misconceptions, we respond, discard the myths. Embrace the orthodoxy: the standard model of cosmology.

In this view of the distance scale, which is widely shared, $\mathrm{H}_{0}$ is the first parameter in a model of an evolving expansion rate. In real experiments, there is a covariance

$\dagger$ http://edd.ifa.hawaii.edu/

$\ddagger$ http://physics.uq.edu.au/ap/cosmicflow/?page_id=14

ब http://db.ipmu.jp/seminar/sysimg/seminar/666.pdf

|l http://www.astron.nl/ jozsa/wnshs/

$\dagger \dagger$ http://physics.uq.edu.au/ap/cosmicflow/?page_id=14

$\ddagger \ddagger$ Australian slang for a rumour, or an erroneous or improbable story 


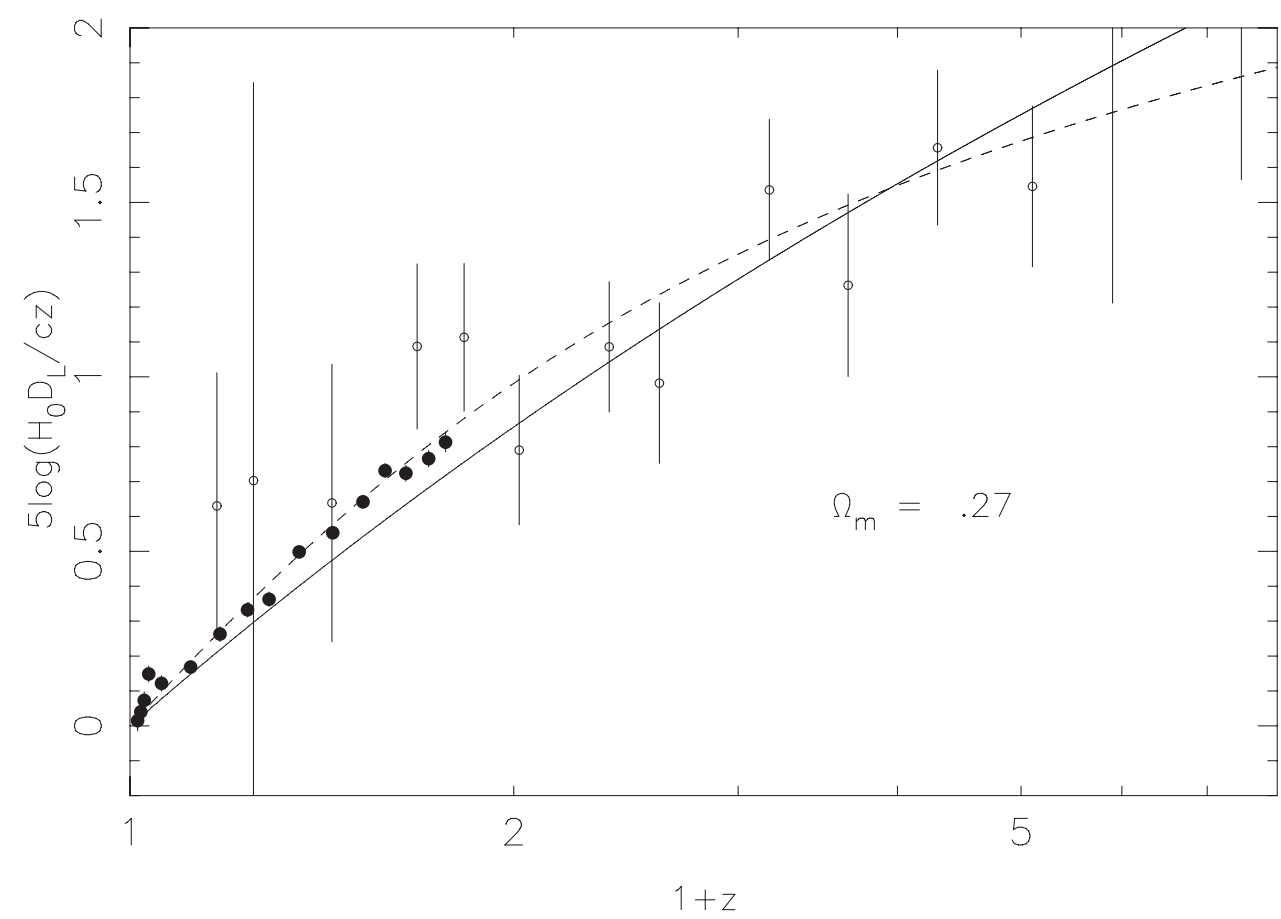

Figure 3. Redshift-distance relation for a steady-state Universe with $\Omega_{\mathrm{M}}=0.27$. This is a curvature-dominated Friedmann equation. Data points: supernovae and gamma-ray bursts. For comparison, the standard model of cosmology with dark energy and zero curvature is shown as a dashed line.

between $\mathrm{H}_{0}$ and other parameters. There are a number of observational constraints on this parameter. They include, but are not confined to, measurements at $z>0$. Variations of density from the mean can be mapped and corrections to a global expansion rate made to local measurements.

A suitable conclusion to a digression on myths is the steady-state Universe. Its fit to supernova data is shown in Fig. 3. To plot the model, I have set $\Omega_{3}=0$ in the polynomial Friedmann equation of Mould (2011) and $\Omega_{0}=\Omega_{\mathrm{M}}$. This corresponds to a constant matter density which does not decrease as $(1+z)^{3}$. The result is a curvature-dominated Universe. In resurrecting the steady-state Universe, one discards conservation of mass. In accepting the standard model of cosmology, one accepts a 70-order-of-magnitude discrepancy between predicted and observed vacuum energy (Martin 2012). However, even if one were to make a choice between these two evils, it is clear from Fig. 3 that the steady-state Universe does not even fit the data for $z<1$, let alone the CMB.

\section{New motivations for an accurate distance scale}

The Wilkinson Microwave Anisotropy Probe model has six free parameters, one of which is the number of relativistic species in the earlier thermal history of the Universe. Steigman (2012) summarizes the evidence that there are four 'neutrinos.' Other evidence is presented by Suyu et al. (2012) and Freedman et al. (2012). If these results are to be raised to the mandatory (Higgs) $5 \sigma$ significance, the Hubble constant will need to 
be measured to $1 \%$. Allan Sandage, to whom this symposium is dedicated, famously described cosmology as the search for two numbers (Sandage 1961). So, he did not subscribe to any of the myths listed in the previous section. Now, there are six numbers. It is a safe prediction that there will be more parameters required to compose a theory of physical cosmology and also that they will steadily become better determined through observations.

\section{Summary}

The Virgo cluster once had an iconic status in the extragalactic distance-scale ladder. In its place, we now have an ensemble of local supercluster distances, connected by a velocity field based on redshift-survey reconstructions. The ladder is now just one of a number of experiments aimed at measuring $\mathrm{H}_{0}$, which is one of six parameters in the current standard model of cosmology. Other experiments are the CMB and baryon acoustic oscillations (BAO). The ladder itself may be bifurcating into the Cepheid-SN Ia ladder and a megamaser-BAO ladder. The former may be the luminosity-distance scale. The latter is an angular-diameter-distance scale. Parameters in the standard model have covariance (degeneracies). This leads to new reasons to constrain $\mathrm{H}_{0}$ to $1 \%$ (e.g. \# neutrinos).

\section{Acknowledgements}

I would like to thank Roger Blandford for hosting a workshop at the Kavli Institute (KIPAC) at Stanford in February 2012, where a number of these ideas were developed. I would also like to recall John Huchra's contributions to the distance scale up to his untimely death in 2010. The study of the dark Universe is supported by the Australian Research Council (ARC) through CAASTRO. $\dagger$ The Centre for All-sky Astrophysics is an ARC Centre of Excellence, funded by grant CE11001020

\section{References}

Aaronson, M. \& Mould, J. 1983, ApJ, 265, 1

Bird, S., Harris, W., Blakeslee, J., \& Flynn, C. 2010, A\&A, 524, 71

Binggeli, B., Sandage, A., \& Tammann, G. 1985, AJ, 90, 1681

Bohringer, H., Briel, U., Schwarz, R., Voges, W., Hartner, G., \& Trumper, J. 1994, Nature, 368, 828

Catinella, B., Kauffmann, G., Schiminovich, D., et al. 2012, MNRAS, 420, 1959

Erdoğdu, P., Lahav, O., Huchra, J. P., et al. 2006, MNRAS, 373, 45

Feldman, H., Watkins, R., \& Hudson, M. 2010, MNRAS, 407, 2328

Ferrarese, L., Côté, P., Cuillandre, J.-C., et al. 2012, ApJS, 200, 4

Freedman, W. L., Madore, B. F., Scowcroft, V., Burns, C., Monson, A., Persson, S. E., Seibert, M., \& Rigby, J. 2012, ApJ, 758, 24

Huchra, J. P., Macri, L. M., Masters, K. L., et al. 2012, ApJS, 199, 26

Lavaux, G., Tully, R. B., Mohayaee, R., \& Colombi, S. 2010, ApJ, 709, 483

Magoulas, C. 2012, Ph.D. thesis, University of Melbourne, Australia

Martin, J. 2012, in: Comptes Rendus de l'Academie des Sciences, in press (arXiv:1205.3365)

Mei, S., Blakeslee, J. P., Côté, P., et al. 2007, ApJ, 655, 144

Mould, J. 2011, PASP, 123, 1030

Mould, J., Aaronson, M., \& Huchra, J. 1980, ApJ, 238, 458

Mould, J. \& Sakai, S. 2008, ApJ, 686, L75

Mould, J. \& Sakai, S. 2009a, ApJ, 694, 1331

$\dagger$ www.caastro.org 
Mould, J. \& Sakai, S. 2009b, ApJ, 697, 996

Sandage, A. 1961, ApJ, 133, 355

Springob, C. M., Masters, K. L., Haynes, M. P., Giovanelli, R., \& Marinoni, C. 2007, ApJS, 172, 599

Steigman, G. 2012, in: Neutrino Physics, Advances in High Energy Physics, in press (arXiv:1208.0032)

Suyu, S., Treu, T., Blandford, R. D., et al. 2012, in: KIPAC workshop on the Hubble constant (arXiv:1202.4459)

Tammann, G. 1999, IAU Symp., 183, 31

Tonry, J. L., Dressler, A., Blakeslee, J. P., Ajhar, E. A., Fletcher, A. B., Luppino, G. A., Metzger, M. R., \& Moore, C. B. 2001, ApJ, 546, 681

Villegas, D., Jordán, A., Peng, E. W., et al. 2010, ApJ, 717, 603

\section{Discussion}

Michael FEAST: Your F350LP observations are going to have problems with calibration and extinction.

JEREMY Mould: We'll work on these in the next six months and also work on photometric simulation. Observations at $V$ and $I$ have also been made.

Giuseppe Bono: Your Coma magnitudes seem to differ from what I would expect.

JEREMY MOULD: Our preliminary Virgo-Coma modulus within the current uncertainties is consistent with the ratio of Coma's redshift and Virgo's flow-corrected redshift. When we have taken the steps I just alluded to, the uncertainty in NGC 4921's distance will drop to perhaps $5 \%$ (I'm guessing) and then we'll see. 